Citing this article: Maatouk, I. \& Jaspal, R. (2020). Barriers to HIV Treatment as Prevention (TasP) in men who have sex with men in the Eastern Mediterranean Region. Journal of Public Health. https://doi.org/10.1093/pubmed/fdz186

\title{
Barriers to HIV Treatment as Prevention (TasP) in men who have sex with men in the Eastern Mediterranean Region Ismael Maatouk, MD, $\mathrm{MPH}^{1}$, Rusi Jaspal, $\mathrm{PhD}^{2}$.
}

\footnotetext{
${ }^{1}$ Dermatology department, Clemenceau Medical Center affiliated with Johns Hopkins International Beirut, Lebanon (ismael.maatouk@ cmc.com.lb);

Faculty of Health \& Life Sciences, De Montfort University, Leicester LE1-9BH, UK (ismael.maatouk@dmu.ac.uk)

${ }^{2}$ Faculty of Health \& Life Sciences, De Montfort University, Leicester LE1-9BH, UK (rusi.jaspal@cantab.net)
}

Running title: TasP in MSM in EMR

Word count of text/abstract: 1471/126

Number of figures: 0

\section{Corresponding author}

Ismael Maatouk, MD, MPH

Department of Internal Medicine - Clemenceau Medical Center affiliated with Johns Hopkins International Beirut, Lebanon (ismael.maatouk@cmc.com.lb);

Faculty of Health \& Life Sciences, De Montfort University, Leicester LE1-9BH, UK (ismael.maatouk@dmu.ac.uk) 
Tel: +9613568968

\begin{abstract}
Treatment as prevention (TasP) refers to the use of antiretroviral therapy to reduce HIV viremia to 'undetectable' levels which in turn eliminates the risk of HIV transmission.

Significant efforts have been made to increase awareness and understanding of TasP in many countries but not in the Eastern Mediterranean Region (EMR) where HIV remains a highly stigmatized condition especially in men who have sex with men. In this editorial, we discuss the effectiveness of TasP and public understanding of it, with a focus on men who have sex with men living in the EMR. We argue that increasing awareness and understanding of TasP could enable the concentrated epidemic in men who have sex with men in the EMR to move closer towards the UNAIDS target of 90-90-90.
\end{abstract}


The significant reduction in HIV viremia to 'undetectable' levels (HIV plasma viral load below 40 copies/ml) in response to effective antiretroviral therapy (ART) removes the risk of HIV transmission [1]. This approach, known as ‘treatment as prevention’ (TasP), has proven to be successful especially in men who have sex with men (MSM) and in other key populations. In view of the demonstrable effectiveness of TasP, significant efforts have been made to increase public awareness and understanding of this approach in Western, industrialized societies like the US and the UK. However, this has not been the case in the Eastern Mediterranean Region (EMR) ${ }^{1}$, in which HIV remains a highly stigmatized condition, which is seldom discussed mainly because of its association with MSM - a stigmatized group in EMR society. In this editorial, we discuss the effectiveness of TasP in major observational studies and current levels of public understanding of, and belief in, TasP, with a focus on MSM in the EMR. We argue that increasing awareness and understanding of TasP in MSM in the EMR could enable the epidemic to move closer towards the UNAIDS target of $90-90-90^{2}$.

\section{TasP: Science \& Public Understanding}

The notion of TasP in MSM has received significant empirical support in major observational studies [1-4]. The HIV Prevention Trials Network (HPTN) 052 study led to the landmark finding that no HIV transmission from the HIV-positive to the HIV-negative primary partner in serodiscordant couples having condomless sex when the HIV-positive partner had a stable suppressed plasma viral load ('suppression' defined by HIV viremia levels < 200 copies/ml) [2]. Moreover, the PARTNER study of 1166 serodiscordant

\footnotetext{
${ }^{1}$ EMR according to WHO includes Afghanistan, Bahrain, Djibouti, Egypt, Iran, Iraq, Jordan, Kuwait, Lebanon, Libya, Morocco, Oman, Pakistan, Qatar, Saudi Arabia, Somalia, Sudan, Syrian Arab Republic, Tunisia, United Arab Emirates and Yemen.

2 The UNAIDS "90-90-90" strategy calls for $90 \%$ of HIV-infected individuals to be diagnosed by $2020,90 \%$ of whom will be on anti-retroviral therapy (ART) and $90 \%$ of whom will achieve sustained virologic suppression. Reaching these targets by 2020 will reduce the HIV epidemic to a low-level endemic disease by 2030.
} 
heterosexual and gay male couples yielded no evidence of any HIV transmissions in the same context [3]. Most recently, data from the Opposites Attract study (358 serodiscordant male couples with high rates of condomless anal sex) revealed no HIV transmissions [4]. These large studies have collectively contributed to the evidence base that people living with HIV (PLHIV) with an undetectable viral load ('undetectable' defined by HIV viremia levels $<40$ copies/ml) cannot transmit HIV sexually.

Consequently, the notion of TasP has been supported by many health agencies, health departments and HIV community advocates [5]. In July 2018, the World Health Organization (WHO) declared that "[The mentioned above] data have led to an increasing consensus that people who have achieved and maintained undetectable viral load cannot transmit HIV sexually to their partners" [6].

Yet, despite this consensus in the scientific community, MSM vary in their levels of understanding and acceptance of TasP. This was demonstrated in a study from Canada (719 MSM) where only $46 \%$ heard of TasP with differences by HIV status (69\% HIV-positive vs. 41\% HIV-negative $(\mathrm{p}<0.0001))$ [7]. In this report, only $6 \%$ of HIV-negative men believed in TasP. In Australia, 13.1\% of MSM surveyed in 2015 believed in TasP [8]. In the UK, a report showed that even MSM living with HIV were ambivalent about the effectiveness of TasP and expressed fears of onward transmission [9]. A large survey of 12,222 MSM in the US showed that a high level of HIV knowledge and low HIV stigma was associated with greater awareness of TasP among HIV-negative people [10].

\section{HIV in the EMR}

According to the WHO, new HIV infections are on the rise in the EMR. The estimated number of new infections increased by $28 \%$ over the past 7 years to reach 36,000 in 2017. This figure represents the highest regional incidence rate [11]. 
While the percentage of the general adult population living with HIV in the Region remained among the lowest globally $(<0.1 \%)$, key populations continue to be disproportionately affected. Ninety-five percent of new HIV infections in the Region are within key populations, i.e. people who inject drugs, MSM and sex workers [12]. Limited access to HIV testing for PLHIV remains the biggest obstacle to the delivery of life-saving antiretroviral therapy in the EMR where only $34 \%$ of PLHIV are aware of their infection [11]. In addition, sexual and injecting partners of PLHIV are at high risk of HIV infection. Extending HIV testing to these population groups would help identify PLHIV who, otherwise, would not know their HIV status [12]. In spite of this, countries of the EMR reported a negligible number of partners of PLHIV who received an HIV test in 2017 [13]. Thus, low testing rates cast doubt on the accuracy of current epidemiological data on the HIV epidemic in the EMR.

Unlike many Western industrialized countries which have achieved, or are close to achieving, 90-90-90 by the end of 2017, the EMR has the lowest performance of all WHO regions, falling short of the 2020 fast-track targets [11]. This can be attributed in part to stigma, discrimination and risk of criminalization, which reduces the reach and effectiveness of HIV prevention programs in the EMR. Protracted humanitarian emergencies and population mobility in many countries of the Region pose an additional challenge for HIV prevention.

\section{Can TasP get us closer to $\mathbf{9 0 - 9 0 - 9 0}$ in the EMR?}

In light of these data, promoting public understanding of TasP (that is, the message of "undetectable=untransmittable") is critical in the EMR, especially in key populations. Awareness and understanding of TasP may lead to: (1) a reduction in HIV stigma; (2) less fear of testing positive and, thus, increased HIV testing; (3) a greater willingness to disclose 
one's HIV status to significant others; (4) and to a better mental health [12]. For instance, in the UK new HIV infections in London have fallen by more than $40 \%$ in recent years and London has already reached 95-98-97 of the global targets [14]. These rates could be achieved with a combination of (1) increasing regular testing in high-risk MSM to facilitate early diagnosis, and (2) early reducing the community viral load through the offer of immediate initiation of antiretroviral treatment [15].

The full potential of TasP in the EMR cannot be exploited if HIV testing for PLHIV remains low (only $34 \%$ of PLHIV are aware of their infection) and if ART coverage is $15 \%$ [11]. The scarce behavioral studies from the EMR in key populations, including MSM, evidence of low HIV testing rates, high levels of condomless anal sex, among many other political, religious and social challenges remain obstacles to achieving 90-90-90 [16].

First, a significant challenge is stigma and discrimination due to the fact that same-sex conduct is illegal in many EMR countries. Homosexuality is punishable by imprisonment, and MSM may experience generalized social stigma and discrimination in society [13]. Second, MSM may experience stigma in from healthcare services which constitutes a barrier in accessing healthcare services and HIV testing [13]. Third, due to stigma, denial and political factors, several EMR governments still assume that HIV is not a significant public health issue in their societies and thus do not dedicate resources to curbing it, instead prioritizing other diseases [13]. This silencing of HIV has led to delayed action to curb the epidemic, allowing HIV incidence to increase and for its syndemics to thrive.

Against this social, political and policy backdrop, it seems unlikely that UNAIDS targets in the EMR will get much closer 90-90-90, unless a bold policy stance on HIV is taken and TasP, as a key prevention method, is embraced. This will require a widespread campaign to raise awareness of the importance of regular and consistent HIV testing in people at risk of HIV and a parallel campaign to educate people about TasP and its role 
within the whole HIV cascade [17]. For TasP to be successful in this area of the world, key stakeholders will need to be convinced of its clinical effectiveness in improving HIV prevention and prognosis, and of its long-term cost effectiveness [17].

Countries that responded pragmatically to the HIV epidemic early on were able to control the spread of infection. The EMR, as a relatively low prevalence region, still has a window of opportunity for curbing its HIV epidemic. HIV testing, ART initiation, and viral load monitoring are needed, together with combination HIV prevention. Ensuring access to available prevention options, and facilitating awareness and understanding of TasP, will not only prevent HIV transmission but also contribute to much needed stigma reduction. This will undoubtedly allow people - both HIV-negative and HIV-positive - to have more open discussions about their sexual health and ultimately to make more informed decisions about their sexual lives.

Acknowledgments: None declared

\section{References}

[1] Cohen MS, Chen YQ, McCauley M, Gamble T, Hosseinipour MC, Kumarasamy N, et al. Antiretroviral therapy for the prevention of HIV-1 transmission. N Engl J Med. 2016; 375:830-9.

[2] Eshleman SH, Hudelson SE, Redd AD, Swanstrom R, Ou S-S, Zhang XC, et al. Treatment as prevention: characterization of partner infections in the HIV prevention trials network 052 trial. J Acquir Immune Defic Syndr. 2017;74:112-6. [3] Rodger AJ, Cambiano V, Bruun T, Vernazza P, Collins S, Van Lunzen J, et al. Sexual activity without condoms and risk of HIV transmission in serodifferent couples when the HIV-positive partner is using suppressive antiretroviral therapy. JAMA. 2016;316:171-81. 
[4] Bavinton BR, Jin F, Prestage G, Zablotska I, Koelsch KK, Phanuphak N, et al. The Opposites Attract Study of viral load, HIV treatment and HIV transmission in serodiscordant homosexual male couples: design and methods. BMC Public Health. 2014;14:917.

[5] McCray E, Mermin JH. Dear Colleague: Information from CDC's Division of HIV/AIDS Prevention. 2017. Available from: https:// www.cdc.gov/hiv/library/dcl/dcl/092717.html [6] World Health Organization. Viral suppression for HIV treatment success and prevention of sexual transmission of HIV. Available from:

http://www.who.int/hiv/mediacentre/news/viral-supression-hiv-transmission/en/

[7] Carter A, Lachowsky N, Rich A, Forrest JI, Sereda P, Cui Z, et al. Gay and bisexual men's awareness and knowledge of treatment as prevention: findings from the Momentum Health Study in Vancouver, Canada. J Int AIDS Soc. 2015; 18:20039. [8] Holt M, Lea T, Schmidt H-M, Murphy D, Rosengarten M, Crawford D, et al. Increasing belief in the effectiveness of HIV treatment as prevention: results of repeated, national surveys of Australian gay and bisexual men, 2013-15. AIDS Behav. 2016; 20:1564-71. [9] Bourne A, Dodds C, Keogh P, Weatherburn P. Non-condom related strategies to reduce the risk of HIV transmission: Perspectives and experiences of gay men with diagnosed HIV. J Health Psychol. 2015 May 6. pii: 1359105315581066. [Epub ahead of print] [10] Rendina HJ, Parsons JT. Factors associated with perceived accuracy of the Undetectable $=$ Untransmittable slogan among men who have sex with men: Implications for messaging scale-up and implementation. Journal of the International AIDS Society 2018, 21:e25055

[11] World Health Organization Eastern Mediterranean Region 2017 estimates. http://www.emro.who.int/world-aids-campaigns/wad2018/index.html [Accessed 18 February 2019] 
[12] UNAIDS. UNAIDS Data 2018. 26 July 2018

http://www.unaids.org/en/resources/documents/2018/unaids-data-2018 [Accessed 7 March 2019]

[13] Hermez, J. Khattabi, H. Sabry, A. Riedner, G. and Hajjeh, R. Achieving the Sustainable Development Goal 3: challenges in HIV testing in the Eastern Mediterranean Region. EMHJ 2017; 23(10): $647-648$

[14] Brown AE, Nash S, Connor N, Kirwan PD, Ogaz D, Croxford S, Angelis D, Delpech VC. Towards elimination of HIV transmission, AIDS and HIV-related deaths in the UK. HIV Med. 2018; Jun 20. [Epub ahead of print]

[15] Nwokolo N, Hill A, McOwan A, Pozniak A. Rapidly declining HIV infection in MSM in central London. Lancet HIV. 2017 Nov;4(11):e482-e483.

[16] Kathryn Risher,1 Kenneth Mayer, Beyer C. The HIV treatment cascade in men who have sex with men, people who inject drugs and sex workers. Curr Opin HIV AIDS. 2015; 10: $420-429$.

[17] Jamieson D, Kellerman SE. The 909090 strategy to end the HIV Pandemic by 2030: Can the supply chain handle it? J Int AIDS Soc. 2016; 19: 20917. 